\title{
Applications of the running 3-min all-out exercise test: an update
}

\begin{abstract}
The running $3 \mathrm{~min}$ all out Exercise Test (3MT) was first used to provide a comprehensive performance assessment of distance runners. Subsequent research has increased the scope of training programs to include a variety of sports utilizing both continuous and non-continuous running (i.e. soccer, rugby). Use of the shuttle running 3MT also has applications for tactical occupations (i.e., law enforcement, military) that require accelerating/decelerating and changing directions. Additionally, with tactical occupations, the use of a running 3MT with an adjustment equation can predict a decline in performance with a specific load from tactical/protective gear. The objective of this review is to provide a brief update on such new applications for the running 3MT.
\end{abstract}

Keywords: Critical speed, high intensity interval training, maximal steady-state, performance modeling
Volume I Issue 6 - 2017

\author{
RobertW Pettitt,' Nathan D Dicks² \\ 'Rocky Mountain University of Health Professions, USA \\ ${ }^{2}$ Department of Health, Nutrition and Exercise Sciences, North
}

Dakota State University, USA

Correspondence: Robert W Pettitt, Rocky Mountain University of Health Professions, USA, Tel I8017346855, Fax 18013752125, Email rpettitt@rmuohp.edu

Received: December 19, 2017 | Published: December 29, 2017
Abbreviations: 3MT, 3-min all-out exercise test; CS, critical speed; D', curvature constant of the speed-time relationship; $t_{\text {LIM }}$, time-limit for sustaining a given running speed and traveling a given distance

\section{Introduction}

The running 3-min all-out exercise test (3MT) estimates the parameters of critical speed (CS) and the curvature constant of the speed-time relationship (D'; pronounced D-prime). ${ }^{1}$ Critical speed is presumed to represent a mechanical measure of the maximal aerobic steady state, demarcating the heavy and severe exercise domains. ${ }^{2}$ Exercise in the severe domain relies upon the continual, timedependent depletion of phosphocreatine, ${ }^{3}$ the time-dependent rise in blood lactate ${ }^{4}$ and evokes a slow component of oxygen uptake that can culminate with attainment of the maximum oxygen uptake at and just prior to the time of exhaustion $\left(\mathrm{t}_{\mathrm{LIM}}\right) .^{5}$ The higher the $\mathrm{D}$ ', the longer distance a person can travel running at speeds exceeding CS before reaching $\mathrm{t}_{\mathrm{LIM}}$. By using the parameters of CS and D', the sport scientist can predict the time or speed one can run different distances (note: typically distances spanning 1,000 to $5,000 \mathrm{~m}){ }^{1}$

The running 3MT has enormous potential for developing racing strategy and is a useful field test for monitoring changes in both endurance and high-intensity exercise capacity. ${ }^{6}$ Although much is written about the utility of the $3 \mathrm{MT}$ to indirectly measure physiological mechanisms governing CS and $\mathrm{D}^{\prime},{ }^{2}$ less is known about the utility of $3 \mathrm{MT}$ for tactical running situations. The purpose of this review is to provide on update on running $3 \mathrm{MT}$ and to discuss developments for the test beyond continuous running events.

\section{Discussion}

The running 3MT involves having the subject run at high-intensity speeds sufficient to exhaust all of $\mathrm{D}^{\prime}$ within a $150 \mathrm{sec}$ duration, whereby the speed of the remaining $30 \mathrm{sec}$ will be equivalent to CS. ${ }^{1}$ The subject should be directed to build up their speed progressively to avoid a muscular injury (e.g. strained hamstring) and then maintain that speed for as long as they can. The timing of the $3 \mathrm{MT}$ can be performed using global positioning sensor (GPS) technology, ${ }^{1}$ video recorded times for specified waypoints, ${ }^{7}$ or by manually recording split times at specified waypoints. ${ }^{8}$ The latter two timing methods are conducive for indoor running, whereas outdoor GPS monitoring allows for testing a large number of subjects in a short period of time (i.e., an entire sports team tested in less than $30 \mathrm{~min}$ ). ${ }^{1}$ The speed of the initial 150sec $\left(\mathrm{S}_{150 \mathrm{~s}}\right)$ is then used to derive $\mathrm{D}^{\prime}$ using: $\mathrm{D}=\left(\mathrm{S}_{150 \mathrm{~s}}-\mathrm{CS}\right)$ 150 sec. $^{1}$ As a concern for pacing exists, we recommend calculating the slope of speed relative to time during last $30 \mathrm{sec}$ of the test (Figure $1){ }^{9}$

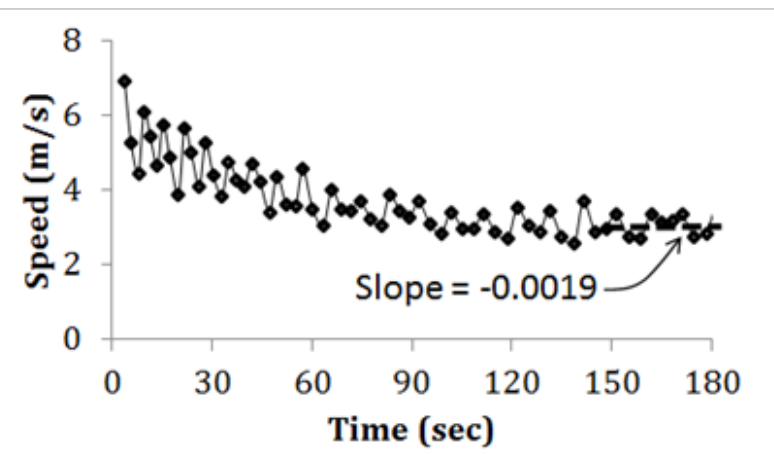

Figure I Example shuttle running 3-min all-out exercise test. Take notice of the more apparent acceleration and deceleration curves during the initial test. The slope is reported to confirm a nadir of speed relative to time (note: the closer the value to zero, the better).

Individualized distance-time and speed-time curves can be derived using data from the running 3MT. The solid lines in Figure 2 depicts the $\mathrm{t}_{\mathrm{LIMs}}$ associated with running 1000,3000 and $5000 \mathrm{~m}$ (left panel) for a representative subject with a CS of $4 \mathrm{~m} / \mathrm{s}$ and a D' of $180 \mathrm{~m}$ along with the corresponding speeds of those performances (right panel). The dashed lines in Figure 2 depicts adjusted $t_{\text {LIs }}$ for $60 \%$ fractional utilization of D' that could be used for high intensity interval training (HIIT). For practical purposes, HIIT bouts utilizing $60 \%$ and $80 \%$ D' can be performed for $4-5$ and 3 sets, respectively, for 90 to $300 \mathrm{sec}$ per interval and a 1:1 and 1:1.5 work: rest ratio, respectively. ${ }^{6}$ 

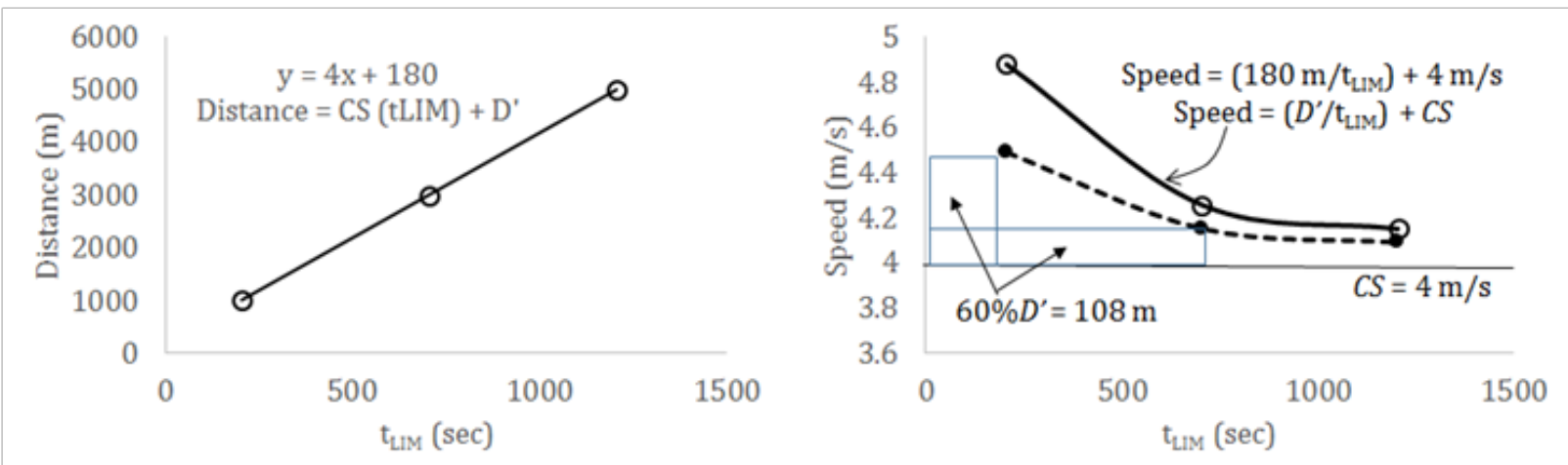

Figure 2 Example distance- $t_{\text {LIM }}$ (left panel) and speed $t_{\text {LIM }}$ relationships for an example subject with a critical speed (CS) of 4 m/s and a D' of I 80 sec. In the left panel, take notice that regression equation for the three different distances and $t_{\text {LIMs }}$ values reveals the CS (slope) and D' ( $y$-intercept). The $t_{\text {LIM }}$ for a given distance may be derived using $t_{\text {IIM }}=\left(D^{\prime}-D^{\prime}\right) / C S$, whereas a longer $t_{\text {IM }}$ for a given distance (D) for a HIIT bout can be derived by multiplying $D^{\prime}$ by the fractional percent (e.g. D'-0.6). In the right panel, take notice of the hyperbolic relationship between speed and $\mathrm{t}_{\mathrm{LIM}}$ relative to CS (solid line), where any speed can be derived with the equation Speed $=\left(D^{\prime} / t_{1 M}\right)+C S$. The speeds corresponding with intervals at $60 \%$ of $D^{\prime}$ are represented by the dashed line. The rectangles represent the $60 \%$ fractional utilization of D', whereby the top, right corner touches the leftward shifted speed-t ${ }_{\text {LIM }}$ curve (dashed line).

The 3MT also is a valid tool for prescribing shuttle running HIIT. ${ }^{9}$ Figure 1 illustrates the acceleration and deceleration trends associated with the turns which are more pronounced during the initial 150 $\mathrm{sec}$ of the test. In that study, $\mathrm{t}_{\text {LIM }}$ values of different shuttle running distances were validated in a similar manner as shown in Figure 2 (i.e., CS and D' from the shuttle 3MT were compared with CS and D' derived from shuttle runs of 3 different distances). When performing the test and running the intervals, it is imperative for the subject to maintain a straight trajectory with full $180^{\circ}$ turns. Equally important, the switch back distance for HIIT bouts should be the same distance as the shuttle running 3MT (i.e., shorter distances with more switch backs will reduce efficiency and therefore distance specific CS). The advantage of the shuttle running method is an interval prescription that is more sport and tactical-specific (i.e., most sports and tactical situations call for repetitive acceleration and deceleration at high speeds with frequent changes in direction). Furthermore, the interval prescription is tailored to the individual athlete/tactical professional replacing the "one size fits all" conditioning programs.

Of particular interest for tactical performance, CS has been evaluated as an alternative to the US Army Physical Fitness Test as a way to assess specific aerobic and anaerobic training needs. ${ }^{10}$ The running $3 \mathrm{MT}$ has been reported to predict combat specific measures in Special Forces soldiers ${ }^{11}$ and therefore the CS concept represents an effect method to prescribing training and monitoring tactical performance. With tactical populations (e.g., military, firefighters, law enforcement), there often is a demand to run with external loads, resulting in greater demands on the aerobic and anaerobic energy systems. ${ }^{12}$ To address the issue of added external load on running performance, Solomonson et al. ${ }^{8}$ reported on the accuracy of an unloaded 3MT to predict running with a specific load, using the adjusted equation $\mathrm{CS}_{\text {loaded }}=\mathrm{CS}_{\text {unloaded }}+(-0.0638 \%$ load $)+0.6982$, where \%load is whole percentage of body mass. Such a model can predict a decline in performance when running with tactical gear, accurate for a range between 15 and $25 \%$ body mass, but also may serve to prescribe HIIT with a load using the modified CS and the appropriate interval equations for fractional utilization of D' (i.e., modeling the dashed line in Figure 2). To date training studies using this model have not been reported and that particular study was confined to continuous high-intensity running.

The above-mentioned studies illustrate the utility of the 3MT and the CS concept. The prescriptions for HIIT are very practical and job-specific for a tactical population (e.g., accelerating/decelerating, changing direction, performing with a load to simulate foot pursuits, tactical movements on the battlefield). Such prescriptions and training regimes not only may increase performance but survivability.

\section{Conclusion}

The running $3 \mathrm{MT}$ was developed as a surrogate measure determining CS and D', two parameters that can be used to predict $\mathrm{t}_{\text {LIMs }}$ associated with various distances and speeds (Figure 2, solid lines). The 3MT also have been used to derive HIIT times (Figure 2, dashed line) and evaluate training-induced adaptations. ${ }^{7}$ Recent studies have validated use of the 3MT for predicting shuttle running performance ${ }^{9}$ along with running with tactical load. ${ }^{8}$ Future investigations are necessary to validate the use the $3 \mathrm{MT}$ to prescribe HIIT for non-continuous running and/or running with a tactical load. Such advances promise to expand the use of the CS concept to a wider range of applications and populations.

\section{Acknowledgments}

None.

\section{Conflict of interest}

Author declares there is no conflict of interest in publishing the article.

\section{References}

1. Pettitt RW, Jamnick N, Clark IE. 3-min all-out exercise test for running. Int J Sports Med. 2012;33(6):426-431.

2. Poole DC, Burnley M, Vanhatalo A, et al. Critical power: An important fatigue threshold in exercise physiology. Med Sci Sports Exerc. 2016;48(11):2320-2334.

3. Jones AM, Wilkerson DP, Menna DF, et al. Muscle metabolic responses to exercise above and below the "critical power" assessed using 31P-MRS. Am J Physiol Regul Integr Comp Physiol. 2008;294(2):R585-R593.

4. Poole DC, Ward SA, Gardner GW, et al. Metabolic and respiratory profile of the upper limit for prolonged exercise in man. Ergonomics. 1988;31(9):1265-1279.

5. Hill DW, Poole DC, Smith JC. The relationship between power and the time to achieve VO2max. Med Sci Sports Exerc. 2002;34(4):709-714. 
6. Pettitt RW. Applying the critical speed concept to racing strategy and interval training prescription. Int $J$ Sports Physiol Perform. 2016;11(7):842-847.

7. Clark IE, West B, Reynolds S, et al. Applying the critical velocity model for an off-season interval training program. J Strength Cond Res. 2013;27(12):1058-1062.

8. Solomonson AA, Dicks ND, Kerr WJ, et al. Influence of load carriage on high-intensity running performance estimation. $J$ Strength Cond Res. 2016;30(5):1391-1396.

9. Saari A, Dicks ND, Hartman ME, et al. Validation of the 3-min all-out exercise test for shuttle running prescription. J Strength Cond Res. 2017.
10. Fukuda DH, Smith AE, Kendall KL, et al. An alternative approach to the Army Physical Fitness Test two-mile run using critical velocity and isoperformance curves. Mil Med. 2012;177(2):145-151.

11. Hoffman MW, Stout JR, Hoffman JR, et al. Critical velocity is associated with combat-specific performance measures in a special forces unit. $J$ Strength Cond Res. 2016;30(2):446-453.

12. Knapik JJ, Reynolds KL, Harman E. Soldier load carriage: historical, physiological, biomechanical and medical aspects. Mil Med. 2004;169(1):45-56. 